\title{
Chronological Endoscopic and Pathological Observations in Russell Body Duodenitis
}

\author{
Atsushi Goto ${ }^{1}$, Takeshi 0 kamoto ${ }^{1}$, Masaharu Matsumoto ${ }^{2}$, Hiroyuki Saito ${ }^{3}$, Hideo Yanai $^{4}$, Hiroshi Itoh ${ }^{5}$ and Isao sakaida ${ }^{1}$ \\ ${ }^{1}$ Department of Gastroenterology and Hepatology, Yamaguchi University Graduate School of Medicine, Ube, Departments of ${ }^{2}$ Gastroenterology \\ and Hepatology and ${ }^{3}$ General Medicine, Hagi Civil Hospital, Hagi, ${ }^{4}$ Department of Gastroenterology and Hepatology, Kanmon Medical Center, \\ Shimonoseki, ${ }^{5}$ Department of Molecular Pathology, Yamaguchi University Graduate School of Medicine, Ube, Japan
}

A 64-year-old man was found to have a nodule in his right lung. He also complained of nausea and abdominal pain during the clinical course. Esophagogastroduodenoscopy revealed a duodenal ulcer associated with severe stenosis and a suspicion of malignancy. However, three subsequent biopsies revealed no evidence of malignancy. The fourth biopsy showed scattered large eosinophilic cells with an eccentric nucleus, leading to a diagnosis of Russell body duodenitis (RBD). RBD is an extremely rare disease, and little is known about its etiology and clinical course. The pathogenesis of RBD is discussed based on our experience with this case.

Clin Endosc 2016;49:387-390

Key Words: Russell body; Russell body duodenitis; Duodenal ulcer

\section{INTRODUCTION}

Russell body duodenitis (RBD) is a rare inflammatory disease characterized by an abundance of polyclonal plasma cells present in the duodenal mucosa. These plasma cells contain intracytoplasmic eosinophilic globules of condensed immunoglobulin, the so-called Russell body. We describe the case of a patient with RBD in whom endoscopic and histopathologic findings were observed chronologically, along with a review of the relevant literature.

\section{CASE REPORT}

The patient is a 64-year-old man. He was found to have a

Received: October 18, 2015 Revised: January 18, 2016

Accepted: January 27, 2016

Correspondence: Atsushi Goto

Department of Gastroenterology and Hepatology, Yamaguchi University Graduate School of Medicine, Minamikogushi 1-1-1, Ube, Yamaguchi, 755-8505, Japan Tel: +81-836-22-2241, Fax: +81-836-22-2303, E-mail: gotomukku@yahoo.co.jp

(cc) This is an Open Access article distributed under the terms of the Creative Commons Attribution Non-Commercial License (http://creativecommons.org/ licenses/by-nc/3.0) which permits unrestricted non-commercial use, distribution, and reproduction in any medium, provided the original work is properly cited. nodule in the right middle lung field on a plain chest radiograph at a local clinic, and was referred to our hospital for detailed examination. A nodule measuring $2 \mathrm{~cm}$ was detected in segment 8 of the right lung on chest plain computed tomography (CT). The morphology of the nodule was lobulated, and cavitation was observed in the interior. Results of smear, culture, and polymerase chain reaction assay from sputum specimens were all negative for tuberculosis. Bronchoscopy was performed on hospital day 9, during which bronchial lavage fluid was collected, and a biopsy of the nodule was performed. Double capsule-like circular objects were positive for periodic acid-Schiff stain and Grocott stain, yielding a diagnosis of pulmonary cryptococcosis. The human immunodeficiency virus (HIV) antibody test was negative. Moreover, the level of the cluster of differentiation (CD) 4 was $570 / \mu \mathrm{L}$, the CD8 level was $655 / \mu \mathrm{L}$, and the $\mathrm{CD} 4 / \mathrm{CD} 8$ ratio was 0.87 , suggesting no evidence of AIDS.

On hospital day 12 , he complained of nausea and right abdominal pain. Abdominal plain CT revealed marked dilatation from the stomach to the duodenal bulb. Esophagogastroduodenoscopy (EGD; day 14) revealed a significant amount of residue and an irregular ulceration with surrounding elevation of the duodenal bulb (Fig. 1A), which had brought about severe stenosis. With the suspicion of duodenal cancer, 

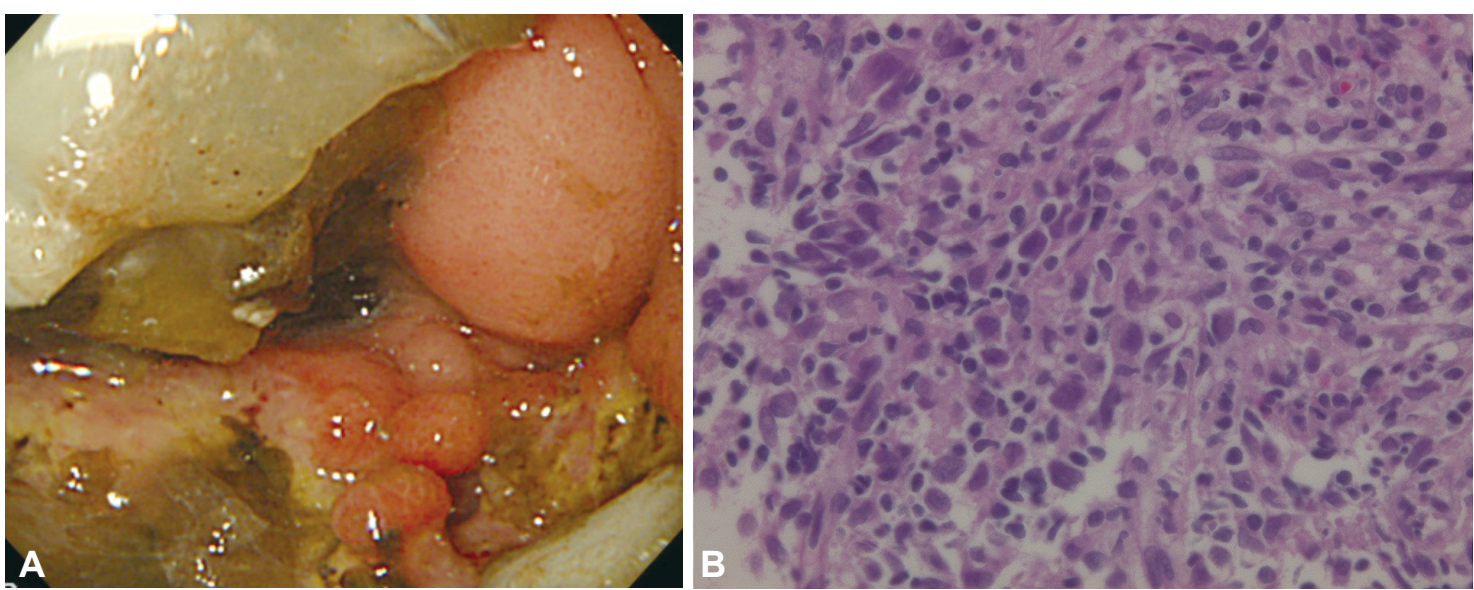

Fig. 1. (A) The first esophagogastroduodenoscopy revealed irregular ulceration in the duodenal bulb. The lumen was obstructed due to severe stenosis. (B) A biopsy was performed from the duodenal ulcer. Inflammatory cell infiltration, necrotic tissue, granulation tissue and large atypical cells were observed (H\&E stain, $\times 200)$.

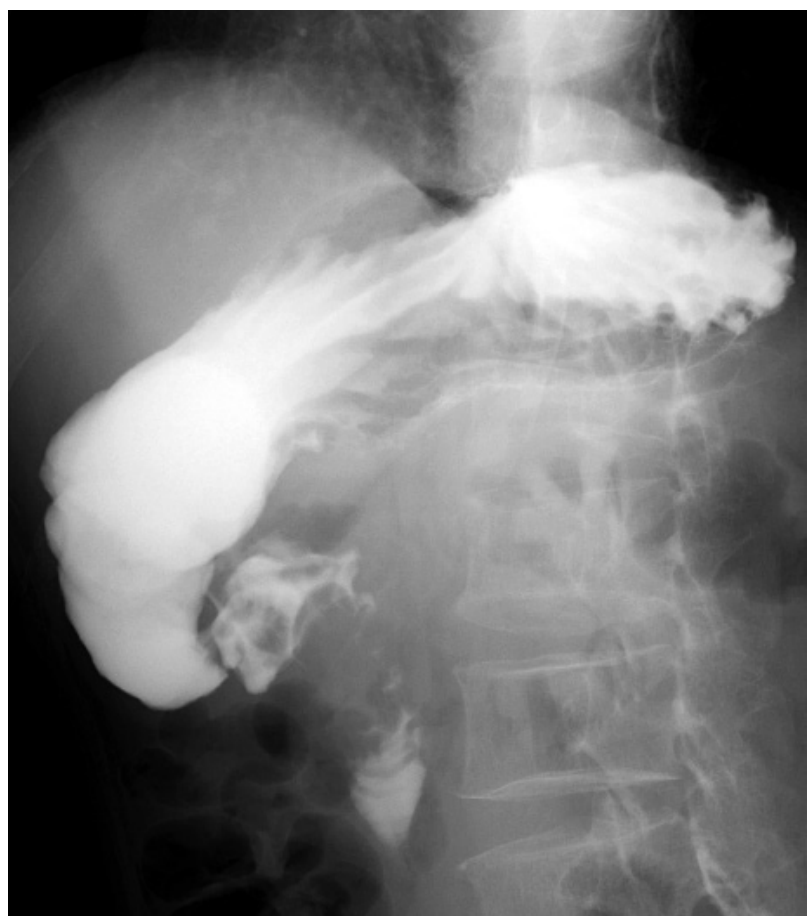

Fig. 2. Contrast-enhanced upper gastrointestinal radiography revealed severe stenosis from the duodenal bulb to the superior duodenal angulus.

biopsy samples were obtained from ulcer edges. Although admixed with various inflammatory cells, necrotic tissues were observed, but no definition of adenocarcinoma cells was found (Fig. 1B). However, since some large atypical cells were admixed with infiltrating inflammatory cells, reexamination was required to rule out undifferentiated carcinoma and malignant lymphoma. A rapid urease test and serum Helicobacter pylori antibody were also negative. The patient was started on omeprazole therapy (40 mg/day). The second EGD (day 21) and the third EGD (day 30) revealed no signs of ulcer healing. Biopsy samples revealed only infiltration of various inflamma- tory cells. There were no findings suggestive of undifferentiated carcinoma and malignant lymphoma. Contrast-enhanced upper gastrointestinal radiography (day 37) revealed severe stenosis from the duodenal bulb to the superior duodenal angle (Fig. 2). Positron emission tomography-CT was performed to screen the whole body for malignant tumors. No significant accumulation of fluorodeoxyglucose was observed in any site except the lesion of pulmonary cryptococcosis in the right lung, and no accumulation was detected in the duodenal bulb. Considering this benign stenosis, we performed a gastrojejunostomy (day 43), and thereafter, oral intake became possible.

The fourth EGD (day 56) revealed that the peripheral redness and edema of the ulcers had become more severe than in the previous findings (Fig. 3A). Biopsy samples revealed dissemination of large cells with eosinophilic cytoplasms and eccentric nuclei in the necrotic/granulation tissue of the duodenal lamina propria (Fig. 3B). The large cells were CD20(-), CD68(-), CD79a $(+)$, and S100(-), and they were $\kappa$ chain $(+)$ or $\lambda$ chain $(+)$, suggesting Russell bodies derived from polyclonal plasma cells (Fig. 3C).

The fifth EGD (day 91) revealed that the ulcers in the duodenal bulb had reduced in size, and some scarring was present (Fig. 4A). Although a biopsy at this time confirmed the presence of a few Russell bodies, the majority had disappeared (Fig. $4 \mathrm{~B})$. The patient is being followed up while continuing the oral administration of a proton pump inhibitor (PPI).

\section{DISCUSSION}

Russell bodies were initially reported by Russell ${ }^{1}$ in 1890 . They are characterized by eosinophilic globules in the cytoplasm of plasma cells. Hsu et al. ${ }^{2}$ showed that Russell bodies originate from distended rough endoplasmic reticulum in 

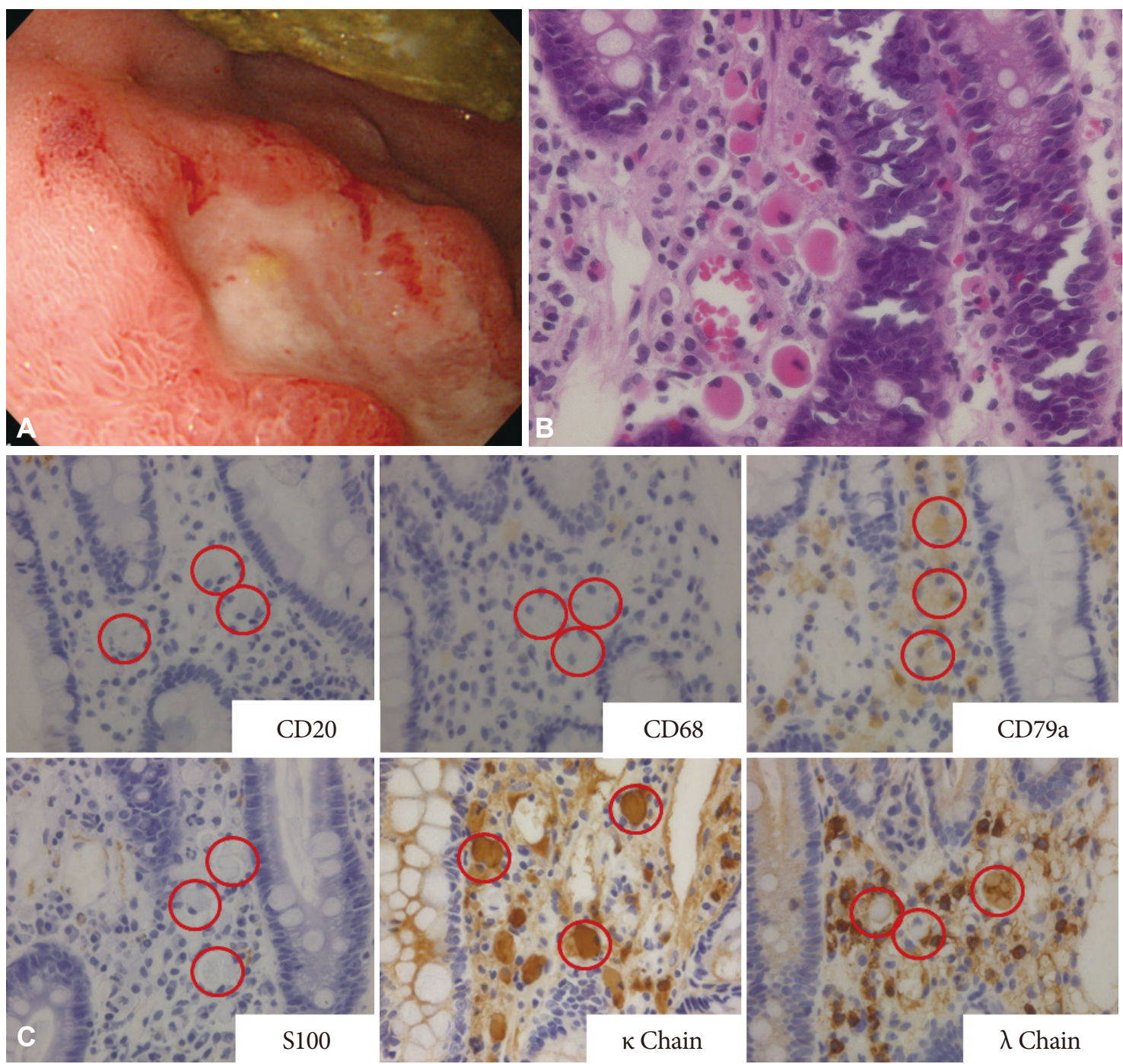

Fig. 3. (A) The fourth esophagogastroduodenoscopy. The peripheral redness and edema of ulcer became more severe than those in the previous findings. (B) The fourth biopsy from the duodenal ulcer revealed dissemination of large cells with eosinophilic cytoplasms and eccentric nuclei in the necrotic/granulation tissue of the duodenal lamina propria (H\&E stain, $\times 200$ ). (C) Immunostaining of the fourth biopsy $(\times 200)$. The red circles indicate Russell body.
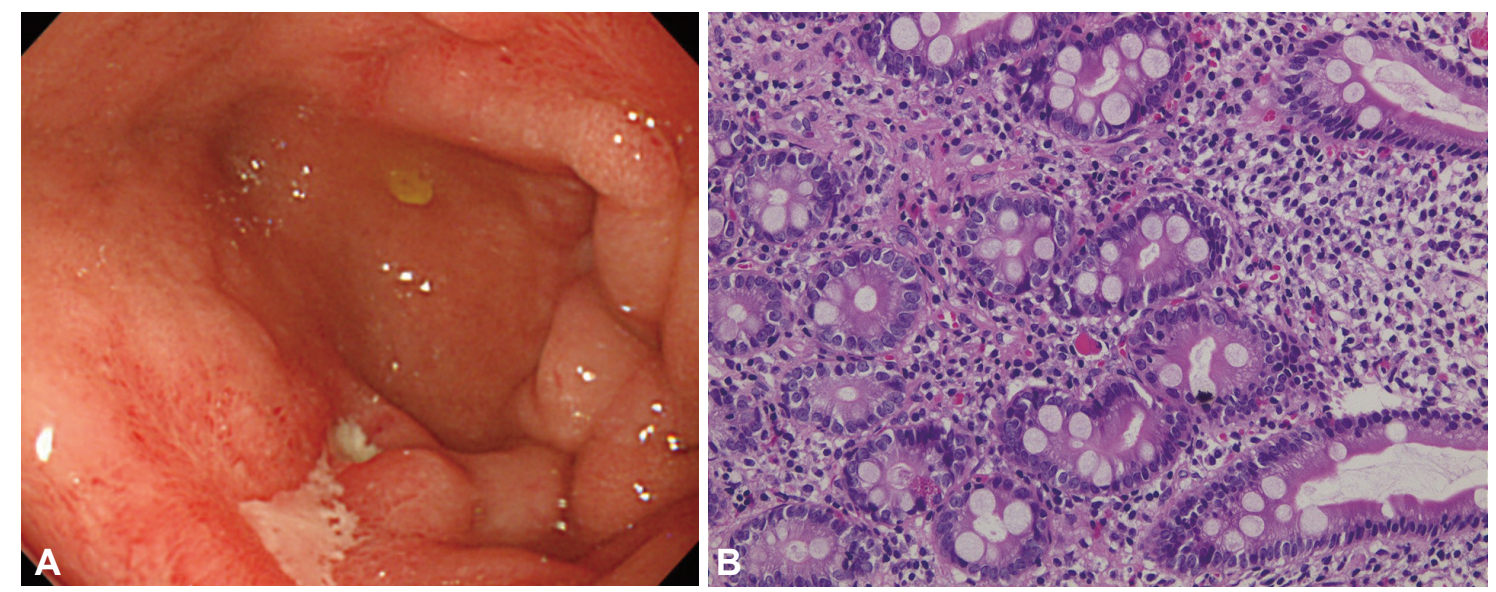

Fig. 4. (A) The fifth esophagogastroduodenoscopy. The ulcers in the duodenal bulb were reduced, some of which were scarred. (B) The fifth biopsy from the duodenal ulcer. Although the biopsy confirmed the presence of a few Russell bodies, the majority of those had disappeared (H\&E stain, $\times 100$ ). 
which immunoglobulin is inhibited from being secreted and condensed. Tazawa and Tsutsumi ${ }^{3}$ first reported Russell body gastritis (RBG) in 1998, in which Russell bodies were found in many plasma cells infiltrating the gastric mucosa. Since then, approximately 20 cases of RBG have been reported. RBD is a rare disease, which Savage et al. ${ }^{4}$ first reported in a patient positive for HIV; only five cases have since been reported.-8 The differential diagnoses include plasmacytoma, mucosa-associated lymphoid tissue lymphoma, and signet ring cell carcinoma. In the present case, because the Russell bodies were derived from plasma cells positive for CD79a and were suggested to be polyclonal by the mixture of $\kappa$ and $\lambda$ chain-positive cells, RBD was diagnosed.

Many patients with RBG are positive for $H$. pylori, which is believed to be associated with the development of RBG. However, only one of the five previously reported cases of RBD was positive for $H$. pylori. The other background diseases were HIV infection in one, ${ }^{4}$ Crohn disease in one, ${ }^{5}$ retroperitoneal metastasis of ureteral cancer in one, ${ }^{6}$ and adenocarcinoma of the ascending colon in one case. ${ }^{8}$ Although the development of Russell bodies is assumed to be associated with chronic inflammation, microorganisms, and immunodeficiency, the causes of their development remain unknown. Although Russell bodies are frequently found in benign tissue adjacent to a malignant tumor, there are also reports suggesting that the production of chemokines in tumor cells is associated with the development of Russell bodies. ${ }^{6.9}$ In the present case, the patient was negative for $H$. pylori and HIV, and there was no evidence of malignancy or immunodeficiency.

We could chronologically observe the endoscopic appearance and histopathological findings of RBD in our patient. When the ulcers were first detected, although severe infiltration of inflammatory cells was observed, RBD was not manifested. Through the aggravation of the ulcers, Russell bodies were discovered on the fourth EGD. Furthermore, the fifth EGD revealed a tendency for the ulcers to heal, and the majority of the Russell bodies had disappeared. From these processes, it was assumed that the Russell bodies were not a cause of the ulcerations, but byproducts secondarily induced by a severe inflammatory state after the development of the ulcers. In the present case, because food residues were always retained in the duodenal bulb, mechanical stimulation might also have been associated with the development of the Russell bodies. Regarding therapeutic responses of Russell bodies, there is a report of patients with RBG who were positive for $H$. pylori, in whom the endoscopic findings improved after $H$. pylori eradication and the Russell bodies disappeared..$^{10}$ The present patient was negative for $H$. pylori and had not undergone $H$. pylori eradication. Atrophic gastritis was also not observed in this patient. His treatment consisted of adminis- tration of a PPI and gastrojejunostomy, which are assumed to have contributed to the resolution of the local inflammation. As for the correlation with the degrees of inflammation at the ulcer site, the Russell bodies would be expected to appear and disappear as the degree of inflammation changed.

The prognosis was favorable with the internal use of a PPI in cases of RBG negative for $H$. pylori. ${ }^{11}$ There have been no published reports of recurrence or malignant transformation during follow-up. Therefore, RBG and RBD are typically found in good disease prognosis. However, it is often difficult to distinguish Russell bodies from plasmacytoma, mucosa-associated lymphoid tissue lymphoma, and signet ring cell carcinoma. Multiple endoscopic observations and biopsies, like that used in the present case, are required to diagnose malignant disease. We will continue to carefully follow this patient.

This was a valuable case in which the clinical and pathological features of RBD, an extremely rare disease, could be compared in the present patient from onset to healing. Further research is required to elucidate the pathogenesis of RBD by studying more cases.

\section{Conflicts of Interest}

The authors have no financial conflicts of interest.

\section{REFERENCES}

1. Russell W. An address on a characteristic organism of cancer. Br Med J 1890;2:1356-1360.

2. Hsu SM, Hsu PL, McMillan PN, Fanger H. Russell bodies: a light and electron microscopic immunoperoxidase study. Am J Clin Pathol 1982;77:26-31.

3. Tazawa K, Tsutsumi Y. Localized accumulation of Russell body-containing plasma cells in gastric mucosa with Helicobacter pylori infection: 'Russell body gastritis'. Pathol Int 1998;48:242-244.

4. Savage NM, Fortson T, Schubert M, Chamberlain S, Lee J, Ramalingam P. Isolated Russell body duodenitis. Dig Dis Sci 2011;56:2202-2204.

5. Paniz Mondolfi A, Samuel M, Kikhney J, et al. Russell body duodenitis: a histopathological and molecular approach to a rare clinical entity. Pathol Res Pract 2012;208:415-419.

6. Takahashi Y, Shimizu S, Uraushihara K, Fukusato T. Russell body duodenitis in a patient with retroperitoneal metastasis of ureteral cancer. World J Gastroenterol 2013;19:125-128.

7. Zhang H, Jin Z, Cui R. Russell body gastritis/duodenitis: a case series and description of immunoglobulin light chain restriction. Clin Res Hepatol Gastroenterol 2014;38:e89-e97.

8. Munday WR, Kapur LH, Xu M, Zhang X. Russell body duodenitis with immunoglobulin kappa light chain restriction. World J Gastrointest Endosc 2015;7:73-76.

9. Bhaijee F, Brown KA, Long BW, Brown AS. Russell body gastroenteritis: an aberrant manifestation of chronic inflammation in gastrointestinal mucosa. Case Rep Med 2013;2013:797264.

10. Karabagli P, Gokturk HS. Russell body gastritis: case report and review of the literature. J Gastrointestin Liver Dis 2012;21:97-100.

11. Del Gobbo A, Elli L, Braidotti P, Di Nuovo F, Bosari S, Romagnoli S. Helicobacter pylori-negative Russell body gastritis: case report. World J Gastroenterol 2011;17:1234-1236. 